\section{Kinase double shot}

\author{
By Tim Fulmer, Senior Writer
}

Based on the binding mechanisms of Gleevec imatinib and Nexavar sorafenib, University of California, San Diego researchers have created a dual kinase inhibitor that selectively hits one of each drug's many targets: RAF and PDGFRB. ${ }^{1}$ Kinagen Inc. has in-licensed the IP and hopes to move a similar compound into the clinic next year.

In the long-running debate on how selective kinase inhibitors should be, the UCSD group's work falls on the narrow end of the spectrum.

The RAS/RAF/MEK/ERK pathway drives the growth and survival of tumor cells and the tumor stroma, which includes any surrounding proangiogenic endothelial cells and pericytes. V-raf-1 murine leukemia (RAF) is important for the survival of tumor cells and endothelial cells during angiogenesis. Platelet-derived growth factor receptor- $\beta$ (PDGFRB) promotes the growth of pericytes, which also lie in the tumor stroma and help maintain the structural integrity of blood vessels.

The UCSD team, led by David Cheresh, vice chair of pathology at the university and cofounder of Kinagen, hypothesized that an inhibitor of both kinases would thus attack cancer on multiple fronts.

The group's first step was studying known X-ray crystal structures that showed the binding mechanisms of Gleevec from Novartis AG and Nexavar from Onyx Pharmaceuticals Inc. and Bayer AG.

Nexavar targets RAF kinases, VEGF receptors, fibroblast growth factor receptor (FGFR), stem cell factor receptor tyrosine kinase (KIT; c-Kit; CD117), FMS-like tyrosine kinase 3 (FLT3; CD135) and platelet-derived growth factor receptors (PDGFRs), ${ }^{2}$ and it is approved to treat hepatocellular carcinoma (HCC) and advanced renal cell carcinoma (RCC). Gleevec also hits multiple kinases including BCR-ABL, PDGFRs and c-Kit, and it is approved for chronic myelogenous leukemia (CML), acute lymphoblastic leukemia (ALL) and gastrointestinal stromal tumors (GIST).

The UCSD team's goal was to design a dual inhibitor that blocked only PDGFRB and RAF. The team hypothesized that such a dual inhibitor would attack both the tumors and their surrounding stroma while avoiding potential side effects associated with drugs that inhibit a broader spectrum of kinases.

According to their respective labels, the most common side effects associated with Nexavar are fatigue, weight loss, diarrhea and rash, whereas Gleevec users can experience edema, nausea, muscle cramps and rash.

The UCSD team's resulting compound inhibited PDGFRB in vitro and also blocked RAF heterodimerization, which is necessary for downstream activation of signaling through mitogen-activated protein kinase kinase (MEK; see Figure 1, "Dual kinase inhibition for cancer").

The compound also reduced the growth of cultured human endothelial cells 25 times more effectively than Nexavar and inhibited growth of

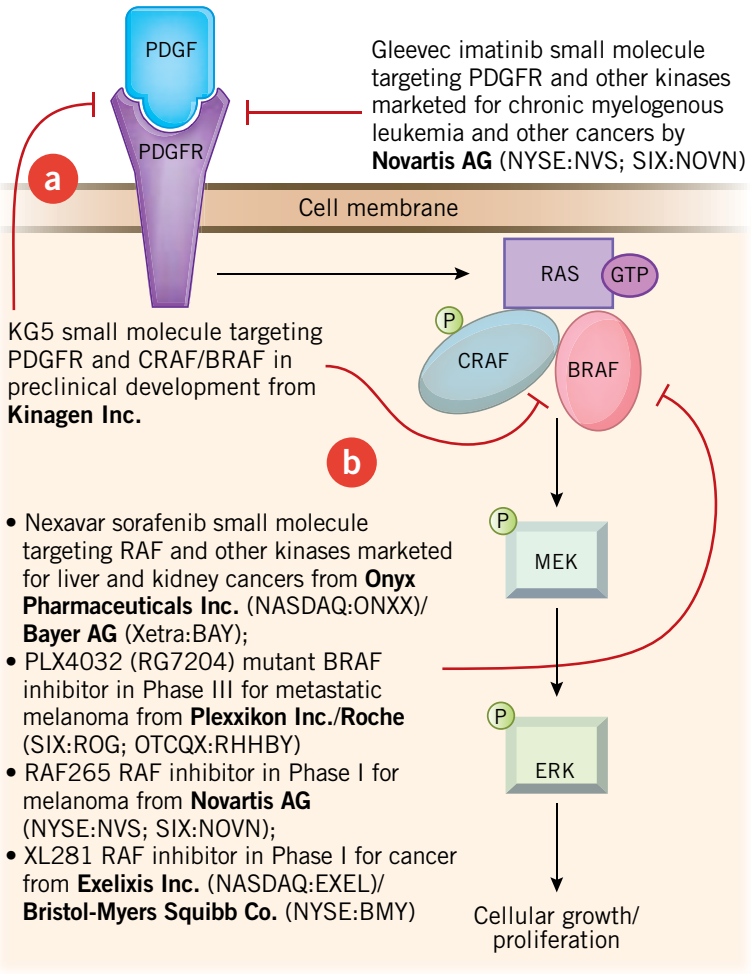

Figure 1. Dual kinase inhibition for cancer. The dual kinase inhibitor developed by researchers at the University of California, San Diego is designed to produce a potent antiangiogenic effect by selectively targeting RAF and PDGFRB. Both kinases are involved in RAS/RAF/ MEK/ERK signaling, a pathway involved in cancer through different means depending on the cell type.

[a] Platelet-derived growth factor receptor- $\beta$ (PDGFRB) is found on pericytes. Activation of this receptor promotes the growth of these cells via RAS/RAF/MEK/ERK signaling. These cells help maintain the structural integrity of blood vessels and enable vessel maturation.

[b] V-raf-1 murine leukemia (RAF) is important for the survival of tumor cells and endothelial cells during angiogenesis and is activated by PDGFRB as well as other growth factor receptors via RAS binding to guanosine triphosphate (GTP). Activated RAS helps recruit RAFfamily molecules to the membrane, triggering the phosphorylation and heterodimerization of CRAF (RAF1) and BRAF. The activated CRAF/BRAF heterodimer induces the downstream kinase mitogenactivated protein kinase kinase (MEK), which in turn triggers mitogenactivated protein kinase 1 (MAPK1; ERK).

By targeting events at both [a] and [b], the dual inhibitor simultaneously blocked angiogenesis and tumor growth in mice and zebrafish. Moreover, the researchers believe the selectivity of such an inhibitor would have safety advantages over broader spectrum, marketed inhibitors such as Gleevec imatinib and Nexavar sorafenib.

cultured vascular smooth muscle cells (VSMCs) better than Gleevec.

In mice induced to undergo neovascularization, the inhibitor completely blocked angiogenesis compared with vehicle control. Also 


\section{Box 1. Targeting the allosteric over the active site.}

Allosteric inhibitors of the cancer target RAF could offer safety advantages over inhibitors that target the kinase's ATP-binding site, according to a trio of new papers.

In an article published in Nature, researchers at Genentech Inc. showed that in some cancer cell lines, inhibitors of v-raf-1 murine leukemia (RAF)'s active site induced a conformational change that actually led to RAF dimerization and activation by RAS. ${ }^{5}$

As a result, even in the presence of an ATP-binding site inhibitor, RAF was still able to trigger the downstream mitogen-activated protein kinase kinase (MEK) and mitogen-activated protein kinase 1 (MAPK1; ERK) signaling that increases tumor growth.
The authors suggested that allosteric inhibitors could avoid the problem by inhibiting the enzyme's function without causing that conformational change.

"Genentech is very interested in exploring RAF allosteric inhibitor strategies that block RAF dimerization and/or activation by RAS," said Shiva Malek, corresponding author on the paper and staff scientist in the company's Department of Biochemical Pharmacology.

In a second paper published in Cell, a team at The Institute of Cancer Research described similar findings.

In cultured cells that expressed mutant forms of RAS, two different RAF ATP-binding site inhibitors increased tumorigenic MEK and ERK signaling. Both molecules did so by driving RAF dimerization and activation. ${ }^{6}$

In the third paper, also published in Nature, researchers at the Memorial Sloan-Kettering Cancer Center, University of California, San Francisco and Plexxikon Inc. showed that ATP-competitive RAF inhibitors blocked ERK signaling in mutant BRAF cells but unexpectedly increased ERK signaling in wild-type BRAF cancer cells by promoting RAF dimerization. ${ }^{7}$ The authors concluded that ATP-competitive RAF inhibitors could be useful for the treatment of tumors driven by BRAF mutations but could have "deleterious effects" in other contexts in which ERK signaling becomes activated, potentially leading to increased tumor growth. compared with vehicle, the compound prevented tumor growth in mouse models of pancreatic cancer and RCC.

The findings were published in the Proceedings of the National Academy of Sciences.

Like Gleevec and Nexavar, the UCSD compound works via allosteric inhibition of the kinase targets. There are two main reasons why this is preferable to directly targeting a kinase's active site and preventing ATP from binding. First, allosteric inhibitors do not have to compete with high ATP levels in the cell to inhibit their kinase targets. Second, the allosteric site is more variable across different kinases than the highly conserved ATP-binding site, thus making it potentially easier to design highly selective inhibitors ${ }^{3}$ (see Box 1, "Targeting the allosteric over the active site").

\section{Moving forward}

Kinagen has exclusively licensed patents covering the dual inhibitor, the scaffold-based chemistry and the cell-based screening platform used in the PNAS study. The biotech also has designed its own dual inhibitor, KG5, that works by the same mechanism as the inhibitor described in the paper.

David Slack, cofounder, president and CEO of Kinagen, said KG5 is undergoing animal toxicology studies and is expected to enter the clinic in $1 \mathrm{H} 11$.

The company's initial Phase I/IIa trial of KG5 is expected to enroll patients with RAF-mutant- and RAS-mutant-positive cancers, including non-small cell lung cancer (NSCLC), colorectal cancer, pancreatic cancer and melanoma. Mutations in the RAF and RAS kinase families can lead to hyperactivation of MEK and mitogenactivated protein kinase 1 (MAPK1; ERK) signaling and can increase cell growth and proliferation, which may trigger tumor formation. ${ }^{4}$

Slack does not think KG5's efficacy will be limited to cancers associated with mutated forms of RAF because the small molecule can bind to wild-type forms of the kinase and also acts on PDGFRB.

The company's strategy contrasts with the approach taken by Plexxikon
Inc., whose RAF inhibitor, PLX4032, selectively targets only the mutant variant V600E of a RAF family member called BRAF. That variant occurs in about $50 \%$ of melanomas. The compound is partnered with Roche and is in Phase III testing to treat metastatic melanoma.

\section{Beyond oncology}

Although Kinagen's lead compound is in the cancer space, the chemistry and screening technology in-licensed from UCSD should be applicable to kinases in other disease areas, noted Cheresh, corresponding author on the PNAS paper.

"Our lab in the UCSD Moores Cancer Center continues to collaborate with Kinagen and is now exploring the potential of using the scaffold-based chemistries and physiological screening platform to develop inhibitors against a range of kinases associated with multiple diseases, including non-oncology indications," he said.

"The Kinagen platform should allow us to generate kinase inhibitors with reduced off-target toxicity and an improved therapeutic index" by precisely controlling selectivity, Cheresh added. "Some marketed kinase inhibitors lack such selectivity and are thus potentially associated with dose-limiting toxicities and other side effects."

Fulmer, T. SciBX 3(8); doi:10.1038/scibx.2010.234

Published online Feb. 25, 2010

\section{REFERENCES}

1. Murphy, E. et al. Proc. Natt. Acad. Sci. USA; published online Feb. 8, 2010; doi:10.1073/pnas.0909299107

Contact: David Cheresh, University of California, San Diego, La Jolla, Calif.

e-mail: dcheresh@ucsd.edu

2. Wilhelm, S. et al. Mol. Cancer Ther. 7, 3129-3140 (2008)

3. Liu, Y. \& Gray, N. Nat. Chem. Biol. 2, 358-364 (2006)

4. Beeram, M. et al. J. Clin. Oncol. 23, 6771-6790 (2005)

5. Hatzivassiliou, G. et al. Nature; published online Feb. 3, 2010; doi:10.1038/nature 08833

Contact: Georgia Hatzivassiliou, Genentech Inc., South San Francisco, Calif.

e-mail: hatzivassiliou.georgia@gene.com 


\section{ANALYSIS/PROSPECTS}

\section{TARGETS \& MECHANISMS}

Contact: Shiva Malek, same affiliation as above e-mail: malek.shiva@gene.com

6. Heidorn, S. et al. Cell; published online Jan. 22, 2010; doi:10.1016/j.cell.2009.12.040

Contact: Richard Marais, Institute of Cancer Research, London, U.K. e-mail: richard.marais@icr.ac.uk

7. Poulikakos, P.I. et al. Nature; published online Feb. 23, 2010; doi:10.1038/nature08902

Contact: Neal Rosen, Memorial Sloan-Kettering Cancer Center, New York, N.Y.

e-mail: rosenn@mskcc.org

\section{COMPANIES AND INSTITUTIONS MENTIONED}

Bayer AG (Xetra:BAY), Leverkusen, Germany

Genentech Inc., South San Francisco, Calif.

The Institute of Cancer Research, London, U.K.

Kinagen Inc., Encinitas, Calif.

Memorial Sloan-Kettering Cancer Center, New York, N.Y.

Novartis AG (NYSE:NVS; SIX:NOVN), Basel, Switzerland

Onyx Pharmaceuticals Inc. (NASDAQ:ONXX), Emeryville, Calif.

Plexxikon Inc., Berkeley, Calif.

Roche (SIX:ROG; OTCQX:RHHBY), Basel, Switzerland

University of California, San Diego, La Jolla, Calif.

University of California, San Francisco, Calif. 